\title{
An Analysis of Player Strategies and Performance in Audio Puzzles
}

\author{
Jaime Carvalho, Luís Duarte, and Luís Carriço \\ LaSIGE \& Department of Informatics, University of Lisbon \\ Edifício C6, Campo-Grande, 1749-016 Lisboa \\ \{jcarvalho, lduarte\}@lasige.di.fc.ul.pt, lmc@di.fc.ul.pt
}

\begin{abstract}
This paper presents the design of a puzzle game for the Android platform also shows a study on puzzle solving strategies across different interaction modalities and showcases a player performance analysis in each identified strategy. Solving puzzles is among the oldest challenges and entertainment activities available to us. However, despite major technological advances, the design of such games has never provided individuals with challenges beyond visual puzzles. We capitalized on this opportunity to tackle the design of puzzles which go beyond visual cues, utilizing sound and vibration feedback as well to offer a fresh challenge to players. Along with the design of this game, our research focused on analyzing puzzle solving strategies applied by users. In particular, this paper details a study in which we analyzed if players apply the same strategy to solve a visual and a audio puzzle. Complementing the strategies analysis on the audio mode, we also present a comparative analysis regarding performance metrics such as completion time, number of moves for completion and the attained score. Results point that players often opt to solve prominent areas first, leaving more abstract zones to the end, independently from the interaction modality involved. Performance analysis tells us that there are suitable strategies to maximize different performance metrics.
\end{abstract}

Keywords: Audio Puzzles, Puzzle Games, Play Strategy.

\section{$1 \quad$ Motivation}

Videogames can be used for various ends, ranging from personal entertainment [2][17], as a catalyst for social interaction [12], as a support tool for teaching and learning process [18] or as an experimental platform for new technologies or design concepts [11]. In the education domain, games are of particular importance for students to develop learning skills which allow them to easily create abstractions of concepts or algorithms [10]. In particular, puzzle games have yielded positive results in such learning process. There are various examples of the usage of puzzle games in distinct areas. Hill [5], Levitin [9][10] and Ross [15] have defended utilizing puzzles and games in general as a motivating factor for a diversity of courses. Ginat [4] has also explored the usage of puzzle games as a catalyst for students in learning environments. Outside the education domain, there has been a deployment of a puzzle 
game to foster communication and collaboration between children with autism spectrum disorder [1]. All these examples are elucidative of the importance of games in general, and in particular puzzle games, for a diversity of domains, improving aspects of people's lives.

In the entertainment domain, and with the proliferation of various types of mobile devices [3], videogames are currently widespread across different platforms [11][12][16]. Furthermore, given the increased computational power [7] and number of features present in modern mobile devices, developers are recurring to different modalities [14] to provide players with alternative challenges which would not have been possible before [6]. Yet, one game type which still lacks proper support is puzzle games. There are a few examples of puzzle games which go beyond the visual version [2], but they are either too simplistic, or are yet to explore the full potential of modern smartphones to provide players with adequate challenges, specifically with puzzle representations which go beyond the traditional figure jigsaw puzzle.

Given the lack of multimodal versions of puzzle games for mobile devices, we envisioned and developed a Multimodal Puzzle Game which allows players to tackle visual or audio puzzles. The game was developed for Android platforms and allows for the full customization of the puzzle challenge, ranging from number of pieces, to help types as well as allowing the selection of any picture or song present in the user's personal library to be a puzzle. Taking inspiration from different puzzle learning strategies and learning environments, we designed a study which aimed at assessing if players use similar strategies for solving multimodal puzzles. In particular we wanted to determine if users tend to prioritize particular puzzle pieces or if they solved the puzzle in the order the pieces are presented to them. Upon identifying a set of solving strategies, we proceeded to a comparative analysis attempting to determine the best approach according to three different metrics: completion time, number of moves for completion and player score. Results provide a clear conclusion as to which strategies yield the best results. The paper is organized as follows: we start by presenting the multimodal puzzle game and all of its features; then we detail our study, present the results o both the identified strategies and on player performance; after, we discuss our findings, draw final conclusions and unveil some of our future research directions.

\section{Multimodal Puzzle Game}

The Multimodal Puzzle Game as its name implies is an application developed for Android platforms which allows users to solve puzzles across different modalities. While puzzle solving games are moderately popular, the available solutions are still rooted to solving visual jigsaw puzzles, the original concept of the physical counterpart of this game. As such, we envisioned a multimodal puzzle game which allows players to not only tackle on picture puzzles, but also on musical ones, in which the main goal is to place segments of a musical piece in the correct order.

The multimodal puzzle game possesses a small selection of features which need to be addressed in detail to fully comprehend the contents of the game, namely the available game modes, configurable options and the game's interface. 


\subsection{Game Modes}

The Multimodal Puzzle Game comprises two game modes: a visual one and an audio mode. The visual mode takes inspiration from traditional physical puzzles in which individuals are required to reconstruct a picture by putting pieces in the appropriate positions. The audio mode has not been so thoroughly explored in both research and videogame industry. In this case it provides a challenge to reconstruct a fragmented song by putting each individual segment in the correct order.

Visual Mode. In visual mode, all image puzzles are square shaped (a limitation to accommodate playing in smartphones). This means puzzles will have $\mathrm{n}^{2}$ number of pieces, where ' $n$ ' is a value configured by the player corresponding to the number of pieces per line. The game provides two approaches towards the visual mode:

- Players can take the challenge of one of three pre-loaded images which come with the game (Fig. 1). These images were created specifically for the Multimodal Puzzle, serving as a default challenge for players.

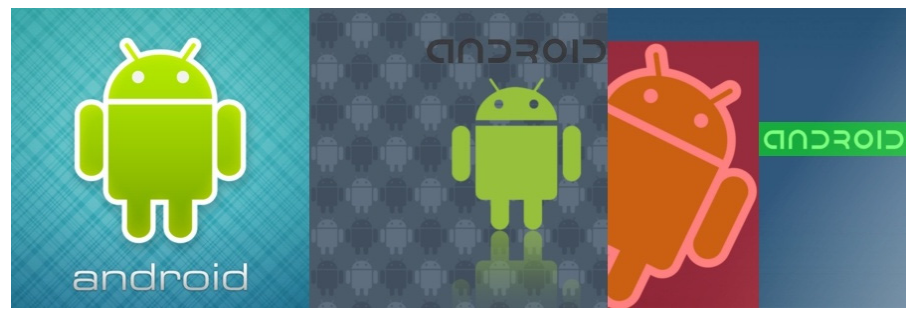

Fig. 1. Default images used for the visual mode puzzle

- The second approach stems from a feature included in the game which allows users to browse images stored in the device. The implication is that players can select any image they desire to solve as a puzzle, effectively broadening the horizon of possible new challenges for the players. If the player picks a non-square shape image, the game stretches the image accordingly to fit the playing area.

Audio Mode. The goal of this game mode is for players to correctly order a musical piece which was divided in a configurable number of segments $\left(\mathrm{n}^{2}\right.$, similarly to the visual puzzle). Each segment is approximately one second long. Similarly to the visual mode, players have two different approaches to play the music puzzle:

- Players can tackle one of three default songs, specifically created for the game. The song contains a repeating calm beat (Fig. 2) which is interrupted by the initial excerpt (first 6 seconds) of Beethoven's $5^{\text {th }}$ Symphony (Fig. 3). The three songs are variations of each other, differing between them in the instant in which the $5^{\text {th }} \mathrm{s}$ excerpt is introduced (in the first variation it is introduced at the $2^{\text {nd }}$ second, in the second variation at the $6^{\text {th }}$ second and in the third variation at the $9^{\text {th }}$ second). 


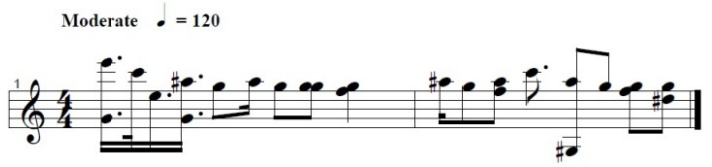

Fig. 2. Beat excerpt created for the default audio puzzle

- Alternatively, players can select any song they have stored in their smartphone and load it to the game in order to complete it as a puzzle. The game automatically segments the song in $\mathrm{n}^{2}$ pieces and then shuffles them. If the player picks a song which is not long enough for the number of pieces established for the puzzle, the game prompts the player if he / she desires to change the puzzle length to a more appropriate one, or if he / she desires to pick a new song. If the song is longer than the available puzzle length (the typical scenario) the initial part of the song is selected to feature in the puzzle.

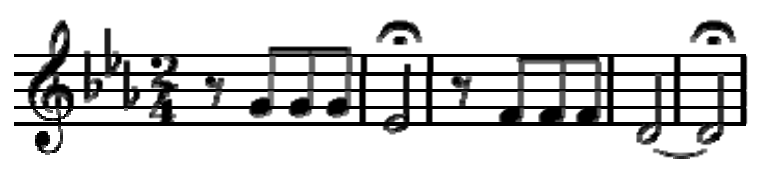

Fig. 3. Beethoven's $5^{\text {th }}$ Symphony initial segment

\subsection{Help Type}

During the course of the game, players have two help types at their disposal: individual and global. In individual mode, players are able to tap one particular square in the unsolved puzzle area to reveal the piece that fits in that place. In the case of the visual puzzle, the individual help displays the image piece belonging to the tapped location; in the case of the music puzzle, the individual help plays the musical segment corresponding to that piece in the puzzle. In global mode, upon tapping the unsolved puzzle area, the whole solution is revealed. This means that in the visual mode, the puzzle figure is shown to the player. When playing the music puzzle, the whole music is reproduced for the player. The help type can be adjusted in the options menu prior to beginning a new puzzle.

\subsection{Rules}

A score based system is used to rank each puzzle solving attempt. Players are awarded 3 points when they place a puzzle piece in the correct position (for the first time per piece only). Positioning a puzzle piece incorrectly deducts one point from the current score. The intent of this system is to force players to think about their actions prior to executing them, avoiding unnecessary penalties for using, for instance, trial \& error strategies. 
Ranks are kept separately for each puzzle type and puzzle size. Games with the same score in the same category (puzzle size and type) are ranked according to the time spent completing the puzzle (with a lower time being better).

\subsection{Interface}

The game's interface can be observed in Fig. 4 (visual mode and audio mode). The main region in the center is the unsolved puzzle area. Here we can see the segmented puzzle and all pieces which still remain to be discovered and the ones which are already placed. Correctly placed pieces keep their original colors, while incorrectly placed ones receive a subtle red transparent layer on top to reveal their current status. A correctly placed piece displays a green transparent layer on top of them for 2 seconds and then assumes its original image fragment (in the case of the visual mode).

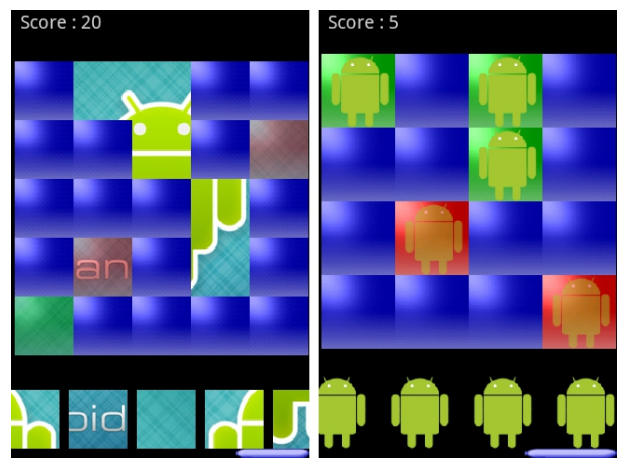

Fig. 4. Multimodal Puzzle Game: Visual Mode and Audio Mode

The lower section of the interface comprises a strip which contains the puzzle pieces. The order of the pieces in this strip is randomly generated prior to each game. In the case of the visual mode, the pieces showcase the image fragment they represent. In the audio mode, each piece has the same visual representation. To access its content, players need to tap once to play the audio segment. To place a puzzle piece in the unsolved area, players tap and drag the piece to the desired position and then let it go to execute the positioning action. During a game, and particularly in large puzzles, players may move a significant number of pieces from the strip to the unsolved puzzle area, causing it to be overcrowded and hindering the comprehension of which pieces are in place and which are not. We implemented a shortcut to make all incorrect pieces return to their original positions in the strip. By double tapping the strip area, the players are able to force all incorrectly played pieces to return to the strip in their original order.

The Multimodal Puzzle Game also comprises a Configuration menu which allows users to set their preferences, such as help type, puzzle size or default image and music library paths. The smartphone's home button opens a quick menu with several shortcuts, namely 'New Game', 'Configuration', quick access to 'Help Type' and 
'Exit Application'. Scores and player preferences are stored in both the Android app and on a XML file for backup.

\section{Experiment}

We conducted an experiment whose goal encompassed identifying which puzzle solving strategies yielded the best performance according to a set of metrics. This experimental period lasted for two weeks, involving three researchers supervising the tests and providing support to subjects as requested.

\subsection{Goals}

The main goal pertained to the identification of which of the assessed strategies yielded the best results according to 3 different parameters: completion time, number of moves for completion and player score. This second overall research goal aims at reinforcing our previous results by providing empirical evidence on the advantages and disadvantages of specific puzzle solving approaches.

\subsection{Research Goals}

In this paper we will focus on performance while solving visual and audio puzzles:

- $\quad$ RG1 - find the most appropriate strategy to solve the audio puzzle based on puzzle completion time.

- $\quad$ RG2 - find the most appropriate strategy to solve the audio puzzle based on total number of movements for puzzle completion.

- $\quad$ RG3 - find the most appropriate strategy to solve the audio puzzle based on the attained score.

\subsection{Variables}

In this study we controlled 4 different variables: the picture puzzle the players had to solve, the audio puzzle needed to be completed, the order in which puzzle pieces were displayed in the strip area of the game, and a fixed puzzle size for each mode. As for the dependent variables, we kept track the order in which pieces were placed in the unsolved area and the order in which each piece category was placed.

\section{Independent Variables}

- Puzzle image - To analyze whether players used the same solving strategy across different images we provided three different images for the players (the Multimodal Puzzle Game default ones: Fig. 1). Albeit three distinct images, their core components are similar in a sense that all include a prominent image of an android character, a small text area and then a simple background. We controlled the usage of the image in the experiment, alternating it between tasks. 
- Puzzle song - Similarly to the previous variable, we provided three different songs for players (the Multimodal Puzzle Game default ones). Again, an excerpt of the background beat is represented in Fig. 2 and is present throughout the whole song. At key instants (at second 2, 6 and 9), the initial segment of Beethoven's $5^{\text {th }}$ Symphony is played. This segment acts as a Type- 1 set piece, the transition between the two beats is considered a Type- 2 set piece and the background beat is considered to belong to Type-3.

- Puzzle strip order - The third independent variable is the order in which puzzle pieces are presented in the strip area. We believe this order might influence the solving order of a puzzle. As such we controlled the way in which pieces are ordered in the strip. 3 variations were implemented: the first scatters the pieces randomly throughout the strip; the second places mostly all Type- 1 and Type- 2 pieces at the end of the strip; the last places mostly all Type- 1 and Type- 2 pieces at the beginning of the strip.

- Puzzle Size - Considering a trade-off between challenge and average time to complete each puzzle (in order to not alienate players) the puzzle size was fixed in 25 pieces for the visual mode and 16 pieces for the audio mode. The discrepancy in puzzle size is due to the amount of time spent in solving the audio mode puzzle which is significantly higher than in the visual mode.

\section{Dependent Variables}

- Game time - This metric will help us understand which strategy yields a faster completion time.

- Total number of moves - Total number of moves is also another way to discern one game from another. By analyzing the number of moves that was taken to complete the puzzle we can reveal the strategy that uses less movement to place pieces into proper place.

- Player score - Once more the rules for score are: +3 points for each piece in the right place, only for the first time, and -1 point for each one on the incorrect place; the score purpose is forcing players to think before acting.

\subsection{Participants}

19 subjects (aged 21 to 27; 18 male, 1 female) participated in this experiment. Individuals were students from different departments in our university. All of them had solved physical puzzles in the past (30\% regularly still solve puzzles) and were proficient with modern smartphones, although the large majority had never played a puzzle game in a smartphone, let alone an audio version.

It is important to say that $40 \%$ of users had musical formation beyond the mandatory given at the high school level (either from specialized courses or through self-learning approaches).

\subsection{Tools and Equipment}

Participants were handed Android smartphones (Samsung Galaxy Mini) to play the game. All devices were previously loaded with the Multimodal Puzzle Game. 


\subsection{Procedure}

The experimental period started with a pre-experiment interview to characterize the subjects (e.g. age, gender, experience with modern smartphones, music theory knowledge, etc.).

The main experiment's procedure was as follows: players were randomly assigned to play either 9 visual mode games or 9 audio mode games. The assignment resulted in 7 subjects playing the visual mode and 12 users playing the audio mode, leading to 63 and 108 play samples respectively, for a total of 171 games.

The 9 mandatory games subjects had to play had the following characteristics:

- Players played 3 games with each one of the 3 default images or songs, depending if they were assigned to the visual or audio mode. The differences between each image and song were disseminated previously in this paper.

- For each image / song players were confronted with a different piece order in the strip area:

- In one of the games Type-1 and Type-2 pieces were randomly scattered throughout the strip.

- In other setting, Type-1 and Type-2 pieces were forcefully put at the end of the strip.

- In the last configuration, Type-1 and Type-2 pieces were forcefully put at the beginning of the strip.

The order of these 9 games was randomly assigned per participant. As an incentive for participation, users were given a download code for the version of the Multimodal Puzzle Game for their Android devices.

\subsection{Results}

Results related with the identification of the most popular strategies applied to solve both the visual and the audio puzzles were published in [19]. In sum, despite more puzzle solving strategies being found in the case of the audio mode, we can state that players primarily recur to 2 strategies when solving puzzles in a mobile device: they attempt to identify the most salient areas of the puzzle (e.g. particular images or segments of a song) and solve those first by prominence order. Secondly they solve the puzzle based on the order pieces are delivered to them even if they can navigate through all pieces. Both these conclusions hold true to the visual and audio modes of the Multimodal Puzzle Game. Nevertheless, we must emphasize that in visual mode only one strategy was clearly prominent and that was solving the puzzle based on the piece prominence in the puzzle. In audio mode, a third approach was found in which players solved the puzzle according to its natural presentation order.

Visual Mode. Given the existence of a single solving strategy (by piece prominence), in Fig. 5 we can see the average time it took to complete each game in visual mode, also as the number of movements and the score achieved. In a way to recall, the game has a total of 25 possible correct moves. For each completion the average amount of time spent rounds 154 seconds, about 2 and a half minutes, and takes 47 moves. The average score obtained is approximately 54 points. 


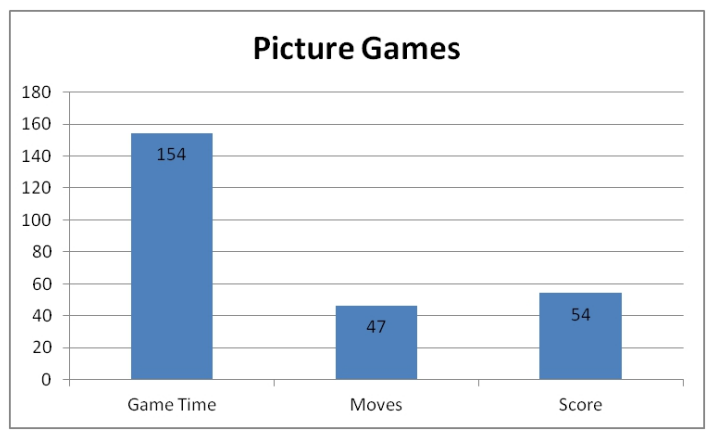

Fig. 5. Visual mode average metrics for time, moves and score

Audio Mode. Results for the audio mode can be observed in Fig. 6, Fig. 7 and Fig. 8. Fig. 6 contains a graph depicting the percentage of games solved according to three identified strategies: by piece category, by the piece strip order and by the puzzle's presentation order (e.g. first row, then second row, etc.). For $9 \%$ of the games we were unable to identify a noticeable strategy.

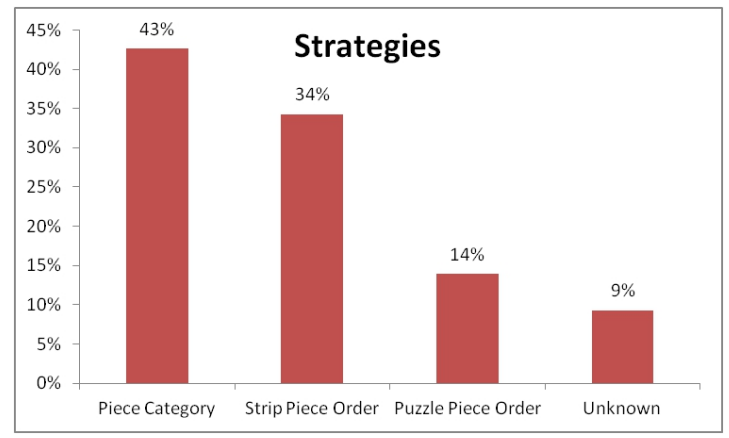

Fig. 6. Audio mode solving strategies distribution

In Fig. 7 we have a simple comparative analysis for all musical puzzle solving strategies in regards to time, movements and score. The first graph shows the average time it took to solve the audio puzzle for each of the strategies; in the center we can see the average number of movements needed to solve the audio puzzle; the last graph holds the average scores reached with each strategy. We also point in each graph the respective averages for the games considered as "unknown", as a way of comparison and as a form of understanding the reasons for their exclusion from the data shown in the graphs above.

We also assessed if players switched strategies over the course of the 9 mandatory games of the experiment: the intent was to check if players adapted over time to the strategies which resulted in higher performance values. The variations of those strategies over time are shown in Fig. 8. In early games (1 and 2) there is an accentuation 


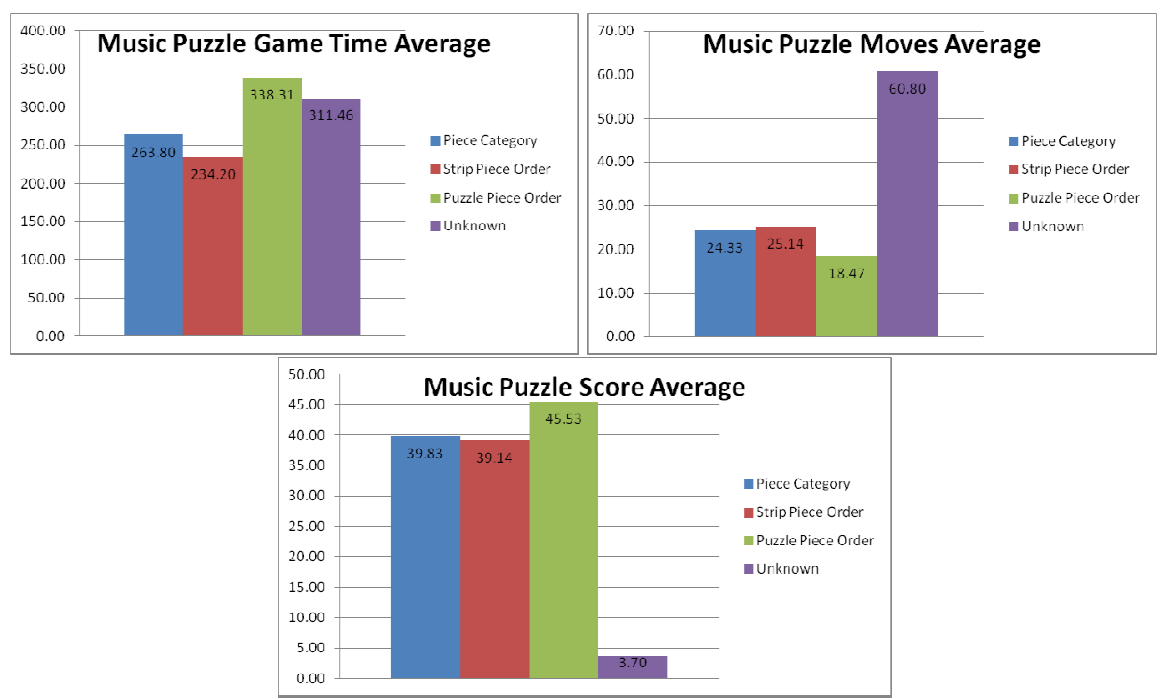

Fig. 7. Audio mode comparative analysis of the audio mode strategies for time, moves and score

of the piece category strategy. From game number 3 to game number 7, we can observe a high variation in the adopted strategies, possibly due to player experimentation with different approaches. However, in the last couple of games we can visualize a confluence towards the strategies which yield the best performance according to the assessed metrics.

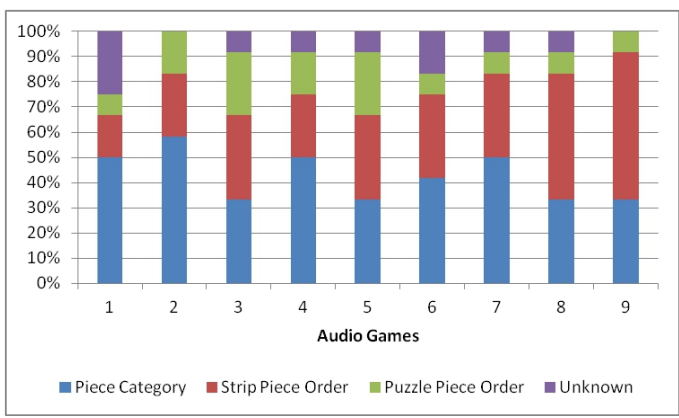

Fig. 8. Audio mode variation strategies over time

\section{Discussion}

We will now discuss these results, taking into consideration the data gathered from visual and audio mode separately. 


\subsection{Visual Mode}

Here, players prioritized solving recognizable pieces immediately, forfeiting background sections of the puzzle to last. These results suggest visual cues are extremely important to solve a puzzle game as individuals will identify them first and attempt to put them together. Given that we attempted to fulfill these research goals with 3 different pictures (albeit and forcefully similar to each other) the gathered empirical data further emphasizes the usage of this strategy.

\subsection{Audio Mode}

Results for the audio mode were not as homogeneous as the ones stemming from the visual mode. By analyzing each game individually we ended up identifying 3 main strategies: piece category prioritization, solving by strip order and solving by puzzle order. Piece category prioritization was the most popular strategy with $43 \%$ of the games following this strategy. Even though a majority of at least $50 \%$ of samples was not reached for this strategy, it is plausible to state RG2 was met for the audio mode. This result emphasizes the importance that players give to prominent regions / segments of a puzzle, which ultimately leads to sharing puzzle solving strategies even across different interaction modalities.

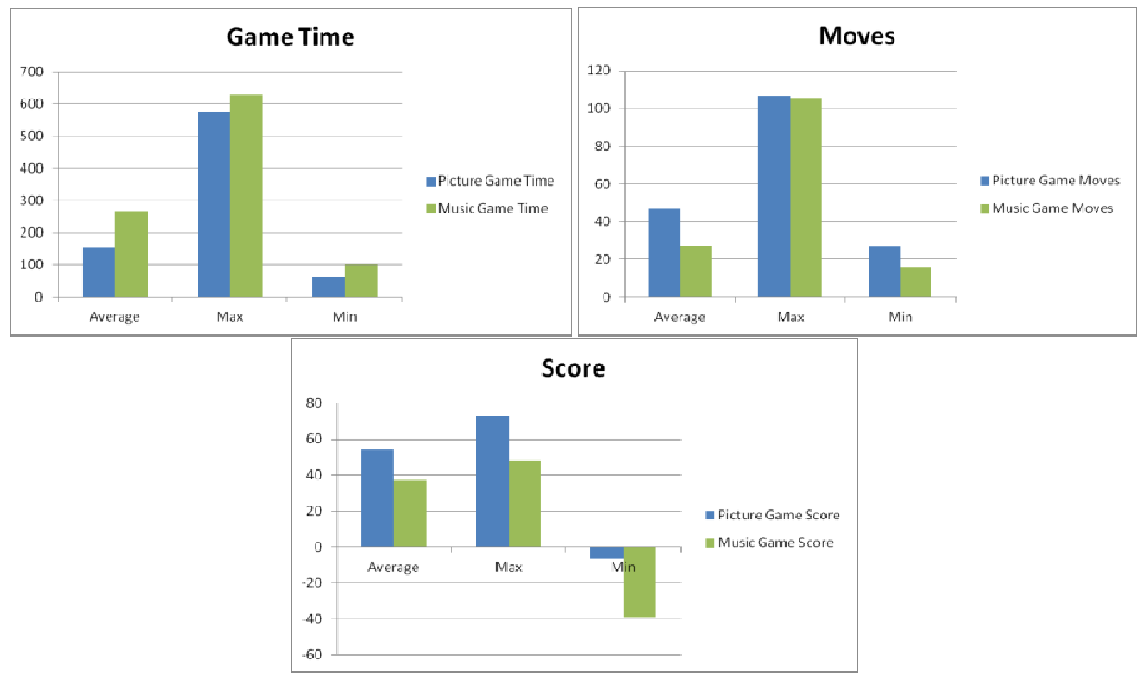

Fig. 9. Comparison between the visual and audio modes for team, moves and score

In order to answer to RG1, RG2 and RG3 we need to analyze Fig. 7. In terms of average time to solve the audio mode it is clear that puzzle piece order is the slowest one with almost 338 seconds (more than five minutes); the fastest is the strip piece order strategy with 234 seconds (nearly four minutes); the piece category strategy stood in the middle with an average of 264 seconds (a little more than four minutes). That being said, the RG4 answer is the strip piece order strategy. The middle graph in 
Fig. 7 helps us figure out which strategy requires less moves to solve a puzzle in audio mode. On average, piece category and strip order strategies are virtually identical with 24 and 25 movements respectively, while puzzle piece order only requires 18 moves, thereby earning the right to RG5. The last metric we considered was the player's score. Here we can verify that on average, once again, piece category and strip order strategies display identical results, with 40 and 39 points respectively. Nevertheless, the answer for our RG6 research goal appears to be the puzzle piece order, providing players with the highest average score at 46 points.

By carefully analyzing the chart in Fig. 8, we can claim that there is no significant convergence towards a single strategy - the distribution for both piece categorization and strip order strategies constantly fluctuates over time; on the other hand the puzzle order strategy obtained a relatively stable number of followers. It is also important to state that four players did not alter their strategy across all 9 games, and the game in which most users altered their strategy was game number 6 . This may have occurred for two reasons: a) due to player fatigue - since the audio version of the experiment lasted for a significant period (averaging more than 40 minutes per subject) we believe a few players were looking for a fast way to end the experiment, and thus changed strategies halfway through it (at around the 22 minute mark of the experiment); b) due to the questionnaire break introduced in game 5 - we asked users to respond to a short survey after game 5, leading us to assume this pause prompted players to explore alternative strategies when they resumed the experiment. In Fig. 9 we present a comparison between visual and audio modes. These charts show that even though the audio puzzle was smaller in size compared to the visual (16 against 25 , respectively), it took much longer to complete. However, the average number of moves performed to complete the audio dropped abruptly when compared to the visual mode. This led us to believe that players in the visual mode recur to "trial and error" approaches more often than in the audio mode.

\section{Conclusions and Future Work}

This paper presented the Multimodal Puzzle Game for Android devices. For the design of this application, we capitalized on the lackluster offer of puzzle games involving challenges beyond visual images. As such we created a game for Android devices which allows players to solve not only image puzzles, but also audio puzzles. The application allows users to tackle on a set of puzzles (both image and audio) created specifically for this game or pick images and songs from the device's own library.

Through this study we can state that there are different strategies for different results, some being more suitable to finish quickly and others to achieve higher scores. The main contribution of this study is the insight on player strategies which can prompt developers and designers alike to build puzzle game UIs to accommodate the users' preferred strategies or those which can maximize player performance.

A cooperative and competitive version of the multimodal puzzle game has already been developed and is currently under usability evaluation. Our intent is to deploy this 
new version and to assess collaborative and competitive puzzle solving strategies, using an experimental approach similar to the one presented in this contribution. Additionally a new modality is also being explored and currently under evaluation: haptics. The intent is to assess the viability of haptic puzzles recurring to vibratory patterns to provide new and innovative challenges to players.

Acknowledgements. This work was funded by FCT, through Individual Scholarship SFRH / BD / 39496 / 2007, through project PTDC/EIA-EIA/103676/2008 (InSIThe) and the Multiannual Funding Programme.

\section{References}

1. Battocchi, A., Pianesi, F., Tomasini, D., Zancanaro, M., Esposito, G., Venuti, P., Ben Sasson, A., Gal, E., Weiss, P.L.: Collaborative puzzle game: a tabletop interactive game for fostering collaboration in children with autism spectrum disorders (ASD). In: Proceedings of the ACM International Conference on Interactive Tabletops and Surfaces, ITS 2009, pp. 197-204. ACM (2009)

2. Diakopoulos, N., Luther, K., Essa, I.: Audio puzzler: piecing together time-stamped speech transcripts with a puzzle game. In: Proceedings of the 16th ACM International Conference on Multimedia, MM 2008. ACM (2008)

3. Duh, H.B.-L., Chen, V.H.H., Tan, C.B.: Playing different games on different phones: an empirical study on mobile gaming. In: Proceedings of the 10th International Conference on Human Computer Interaction with Mobile Devices and Services, MobileHCI 2008. ACM (2008)

4. Ginat, D.: Elaborating heuristic reasoning and rigor with mathematical games, pp. 32-36. ACM (December 2007)

5. Hill, J.M.D., Ray, C.K., Blair, J.R.S., Carver Jr., C.A.: Puzzles and games: addressing different learning styles in teaching operating systems concepts. In: Proceedings of the 34th SIGCSE Technical Symposium on Computer Science Education, SIGCSE 2003, pp. 182-186. ACM, New York (2003)

6. Hoggan, E., Brewster, S.A.: Crosstrainer: testing the use of multimodal interfaces in situ. In: Proceedings of the 28th International Conference on Human Factors in Computing Systems, CHI 2010, pp. 333-342. ACM, New York (2010)

7. Korhonen, H., Koivisto, E.M.I.: Playability heuristics for mobile multiplayer games. In: Proceedings of the 2nd International Conference on Digital Interactive Media in Entertainment and Arts, DIMEA 2007, pp. 28-35. ACM, New York (2007)

8. Lee, S., Zhai, S.: The performance of touch screen soft buttons. In: Proceedings of the 27th International Conference on Human Factors in Computing Systems, CHI 2009, pp. 309-318. ACM, New York (2009)

9. Levitin, A.: Analyze that: puzzles and analysis of algorithms. In: Proceedings of the 36th SIGCSE Technical Symposium on Computer Science Education, SIGCSE 2005, pp. 171-175. ACM (2005)

10. Levitin, A., Papalaskari, M.-A.: Using puzzles in teaching algorithms. In: Proceedings of the 33rd SIGCSE Technical Symposium on Computer Science Education, SIGCSE 2002, pp. 292-296. ACM, New York (2002) 
11. Bell, M., Brown, B., Hall, M., Sherwood Matthew Chalmers, S., Barkhuus, L., Tennent, P.: Gaming on the edge: Using seams in pervasive games. In: PerGames 2005 Proceedings, Munich, Germany, pp. 11-18 (2005)

12. Paelke, V., Reimann, C.: Vision-based interaction - a first glance at plazing MR games in the real-world around us. In: PerGames 2005 Proceedings, Munich, Germany, pp. 92-97 (2005)

13. Poupyrev, I., Maruyama, S.: Tactile interfaces for small touch screens. In: Proceedings of the 16th Annual ACM Symposium on User Interface Software and Technology, UIST 2003, pp. 217-220. ACM (2003)

14. Reufer, T., Panknin, M., Geiger, C.: Sensodroid: multimodal interaction controlled mobile gaming. In: Proceedings of the 13th International Conference on Humans and Computers, HC 2010, Fukushima-ken, Japan, pp. 32-36 (2010)

15. Ross, J.M.: Guiding students through programming puzzles: value and examples of java game assignments, vol. 34, pp. 94-98. ACM (December 2002)

16. Sedano, C.I., Laine, T.H., Vinni, M., Sutinen, E.: Where is the answer?: the importance of curiosity in pervasive mobile games. In: Proceedings of the 2007 Conference on Future Play, Future Play 2007, New York, NY, USA, pp. 46-53 (2007)

17. Holleis, A.W.P., Kranz, M., Schmidt, A.: Playing with the real world. In: PerGames 2005 Proceedings, Munich, Germany, pp. 43-50 (2005)

18. del Blanco, A., Torrente, J., Marchiori, E.J., Martinez-Ortiz, I., Moreno-Ger, P., Fernandez-Manjon, B.: Easing assessment of game-based learning with <e-adventure $>$ and LAMS. In: Proceedings of the Second ACM International Workshop on Multimedia Technologies for Distance Learning, MTDL 2010, pp. 25-30. ACM, New York (2010)

19. Carvalho, J., Duarte, L., Carriço, L.: Puzzle Games: Players Strategies Across Different Interaction Modalities. In: Proceedings of Fun \& Games 2012 (2012) 